\title{
DNA methylation of hypertension-related genes is influenced by the MTHFR 677TT genotype and riboflavin supplementation
}

\author{
Sophia Amenyah $^{1,2}$, Mary Ward ${ }^{1}$, Amy McMahon ${ }^{1}$, Jennifer Deane ${ }^{2}$, Helene McNulty ${ }^{1}$, \\ Catherine F. Hughes ${ }^{1}$, J.J. Strain ${ }^{1}$, Geraldine Horigan ${ }^{1}$, John Purvis ${ }^{3}$, Colum P. Walsh ${ }^{2}$ and \\ Diane J. Lees-Murdock ${ }^{2}$ \\ ${ }^{1}$ Nutrition Innovation Centre for Food and Health, Ulster University, Coleraine, United Kingdom, \\ ${ }^{2}$ Genomics Medicine Research Group, Ulster University, Coleraine, United Kingdom and \\ ${ }^{3}$ Department of Cardiology, Altnagelvin Area Hospital, Londonderry, United Kingdom
}

\begin{abstract}
Introduction: The C677T polymorphism in the folate metabolising enzyme methylenetetrahydrofolate reductase (MTHFR) is associated with hypertension. Riboflavin acts as a cofactor for MTHFR in one-carbon metabolism which generates methyl groups for utilisation in important biological reactions such as DNA methylation. Supplementation with riboflavin has previously been shown to lower blood pressure in individuals with the MTHFR 677TT genotype. The mechanism regulating this gene-nutrient interaction is currently unknown but may involve aberrant DNA methylation which has been implicated hypertension.
\end{abstract}

Objectives: The aims of this study were to examine DNA methylation of hypertension-related genes in adults stratified by $M T H F R$ C677T genotype and the effect of riboflavin supplementation on DNA methylation of these genes in individuals with the MTHFR 677TT genotype.

Materials and Methods: We measured DNA methylation using pyrosequencing in a set of candidate genes associated with hypertension including angiotensin II receptor type 1 (AGTRI), G nucleotide binding protein subunit alpha 12 (GNA12), insulin-like growth factor 2 (IGF2) and nitric oxide synthase 3 (NOS3). Stored peripheral blood leukocyte samples from participants previously screened for the MTHFR C677T genotype who participated in targeted randomised controlled trials $(1.6 \mathrm{mg} / \mathrm{d}$ riboflavin or placebo for 16 weeks) at Ulster University were accessed for this analysis $(\mathrm{n}=120)$.

Results: There were significant differences in baseline average methylation between MTHFR CC and TT genotypes at NOS3 ( $\mathrm{p}=$ $0.026)$ and AGTRI $(\mathrm{p}=0.045)$ loci. Riboflavin supplementation in the TT genotype group resulted in altered average methylation at IGF2 ( $\mathrm{p}=0.025)$ and $\mathrm{CpG}$ site-specific alterations at the AGTRI and GNA12 loci.

Conclusion: DNA methylation at genes related to hypertension were significantly different in individuals stratified by MTHFR genotype group. Furthermore, in MTHFR 677TT genotype individuals, there were concurrent alterations in DNA methylation at genes linked to hypertension in response to riboflavin supplementation. This is the largest study to date to demonstrate an interaction between DNA methylation of hypertension-related genes and riboflavin supplementation in adults with the MTHFR 677TT genotype. Further work using a genome-wide approach is required to better understand the role of riboflavin in altering DNA methylation in these genetically at-risk individuals.

\section{Conflict of Interest}

There is no conflict of interest 